\title{
Cis elements and potential trans-acting factors for the developmental regulation of the Phaseolus vulgaris $\mathrm{CHS} 15$ promoter
}

\author{
Grant S. Hotter ${ }^{1,3}$, Jan Kooter ${ }^{2,4}$, Ian A. Dubery ${ }^{5}$, Christopher J. Lamb ${ }^{2}$, Richard A. Dixon ${ }^{1}$ and \\ Maria J. Harrison ${ }^{1, *}$ \\ ${ }^{1}$ Plant Biology Division, The Samuel Roberts Noble Foundation, PO Box 2180, Ardmore, OK 73402, \\ USA (* author for correspondence); ${ }^{2}$ Plant Biology Laboratory, Salk Institute for Biological Studies, 10010 \\ North Torrey Pines Road, La Jolla, CA 92037, USA ; ${ }^{3}$ Current address: Molecular Genetics Unit, \\ Department of Microbiology and Genetics, Massey University, Palmerston North, New Zealand; ${ }^{4}$ Current \\ address: Vrije Universiteit, BioCentrum Amsterdam, IMBW, Dept. Genetics, De Boelelaan 1087, 1081 HV \\ Amsterdam, Netherlands; ${ }^{5}$ Department of Biochemistry, R.A.U. University, Auckland Park, South Africa
}

Received 7 December 1994; accepted in revised form 18 May 1995

Key words: chalcone synthase, cis elements, Phaseolus vulgaris, seed development

\begin{abstract}
A nuclear factor (SBF-1) has previously been identified in Phaseolus vulgaris L. (bean) suspension cell nuclear extracts that binds in vitro to three DNase I-footprinted elements (SBF-1 boxes I, II, and III, $5^{\prime}$ to $3^{\prime}$ ) in the $5^{\prime}$ region of the bean CHS15 (chalcone synthase) gene promoter. To define the functional role of the three SBF-1 boxes in development, we examined transgenic tobacco plants carrying a series of nested $C H S 15$ promoter- $\beta$-glucuronidase (GUS) fusions for GUS activity by histochemical staining. We show that the CHS15 promoter deleted to position -173 and lacking all three SBF-1 boxes directs the same qualitative pattern of expression in initiating lateral roots and in developing seeds as the full length promoter (-326). Thus, activation of expression in these organs is mediated by sequence elements located downstream of the three SBF-1 boxes. However, specific deletions within the -326 to -173 region modulate expression. Thus, deletion of box II abolishes GUS activity in initiating lateral roots. Further deletion of box III fails to restore expression but subsequent deletion of an additional 43 bp to position -173 re-establishes expression. We show that sequence-specific DNA-binding activities consistent with these results are present in nuclear extracts of bean roots and seeds. These studies reveal cis elements within the CHS 15 promoter, and potential trans factors, that permit organ- and tissue-specific developmental patterns of regulation to be combined with a flexible response to environmental cues.
\end{abstract}

\section{Introduction}

Differential transcriptional activation of plant genes in response to spatial, developmental, and environmental cues is ultimately mediated by the binding of transcription factors to specific se- quence elements present in the promoter of the transcribed gene. We are interested in defining the transcriptional mechanisms by which the diverse range of spatial, developmental, and environmental cues impacting a plant are interpreted to achieve regulated transcriptional activation of 
genes in the phenylpropanoid biosynthetic pathway.

Chalcone synthase (CHS) catalyzes the first committed step in the phenylpropanoid pathway leading specifically to the synthesis of flavonoids, isoflavonoids and related compounds. Subsets of these compounds are important as low molecular weight antimicrobial phytoalexins, as pigments, in reproduction, and in protection from UV light [9]. Flavonoids have also been implicated in the regulation of polar auxin transport [13], and serve as signals for the establishment of legumeRhizobium symbiosis [6, 22, 23].

In French bean (Phaseolus vulgaris L., henceforth 'bean') CHS comprises a gene family of at least seven members, for which differential enzyme biosynthesis is regulated at the transcriptional level in response to a wide range of external and internal cues [25]. Individual members of the gene family are differentially transcribed in a tissue- and development-specific manner, although overlap in expression is observed; hence, the overall pattern of CHS expression reflects the sum of differential transcriptional activation of the individual CHS genes [28].

The promoter regions of two members of the bean CHS gene family, CHS 8 and $C H S 15$, have been previously examined. In transgenic tobacco, a $1.4 \mathrm{~kb} C H S 8$ promoter: $\beta$-glucuronidase (GUS) reporter gene is activated in the inner epidermal cells of petals, and in root and lateral root meristems [28]. The CHS 8 promoter is inducible in leaves by both abiotic and biotic elicitors, including UV light, wounding, mercuric chloride, fungal elicitor, or infection with Pseudomonas syringae $[3,28,30]$. In contrast, a 490 bp CHS15 promoter:GUS reporter gene is activated in transgenic tobacco by UV light and mercuric chloride, but not by $P$. syringae [30]. The analysis of deleted forms of either promoter, to functionally dissect the individual promoter elements controlling tissue-specific expression, have not yet been performed in planta.

Most functional studies involving the CHS15 promoter have been performed in electroporated soybean or alfalfa protoplasts, and have shown that the promoter is inducible by either fungal elicitor or glutathione $[2,5,10,17]$. These studies, together with gel-retardation assays and invitro DNase I footprinting $[10,17,32]$ have defined several functional regulatory elements that serve as the binding sites for bean and/or (equivalent) alfalfa nuclear proteins (Fig. 1). These elements include a G-box (CACGTG) and three H-boxes (CCTACC[N] $]_{7} \mathrm{CT}$ ) [32], which, in combination, are necessary and sufficient for stimulation of transcription by the phenylpropanoid pathway intermediate trans-p-coumaric acid $[20,21]$ and are also implicated in activation of the promoter in response to fungal elicitor and glutathione.

Distal to the $\mathrm{H}$ - and G-boxes are three sequence elements, designated box I, II, and III which are involved in the quantitative induction of CHS15 transcription in both elicited and unelicited cells $[5,10]$. These three boxes are footprinted in vitro with bean and alfalfa nuclear extracts $[10,17]$ and each bears either one or two copies of the consensus motif GGTTAA(A/T)(A/ $\mathrm{T})(\mathrm{A} / \mathrm{T})$ [17] (Fig. 1). The first six bases of this consensus motif are identical to the GT-1 recognition motif GGTTAA [8]. A bean nuclear factor, designated SBF-1 (Silencer Box Factor-1) due to the silencing effect of the box I, II, III region on chloramphenicol acetyltransferase (CAT) reporter gene activity in electroporated soybean protoplasts [5], has been identified that binds in vitro to each of box I, II, and III, with highest affinity for box III [17]. Phosphorylation of SBF-1 is required for binding activity, and may also be required to maintain stable binding in a preformed SBF-1/DNA complex [11].

Initial attempts to obtain a cDNA clone for SBF-1 have not been successful and are further hindered by a lack of knowledge of the specific expression patterns of the CHS15 gene in planta. In particular, information regarding the precise tissues in which CHS15 is expressed and also in which the distal region of the promoter containing the three SBF-1 boxes can be demonstrated to influence CHS 15 expression, would clearly be beneficial for the cloning of this factor. Due to the high level of sequence identity of the individual CHS genes within the bean multigene family it is 


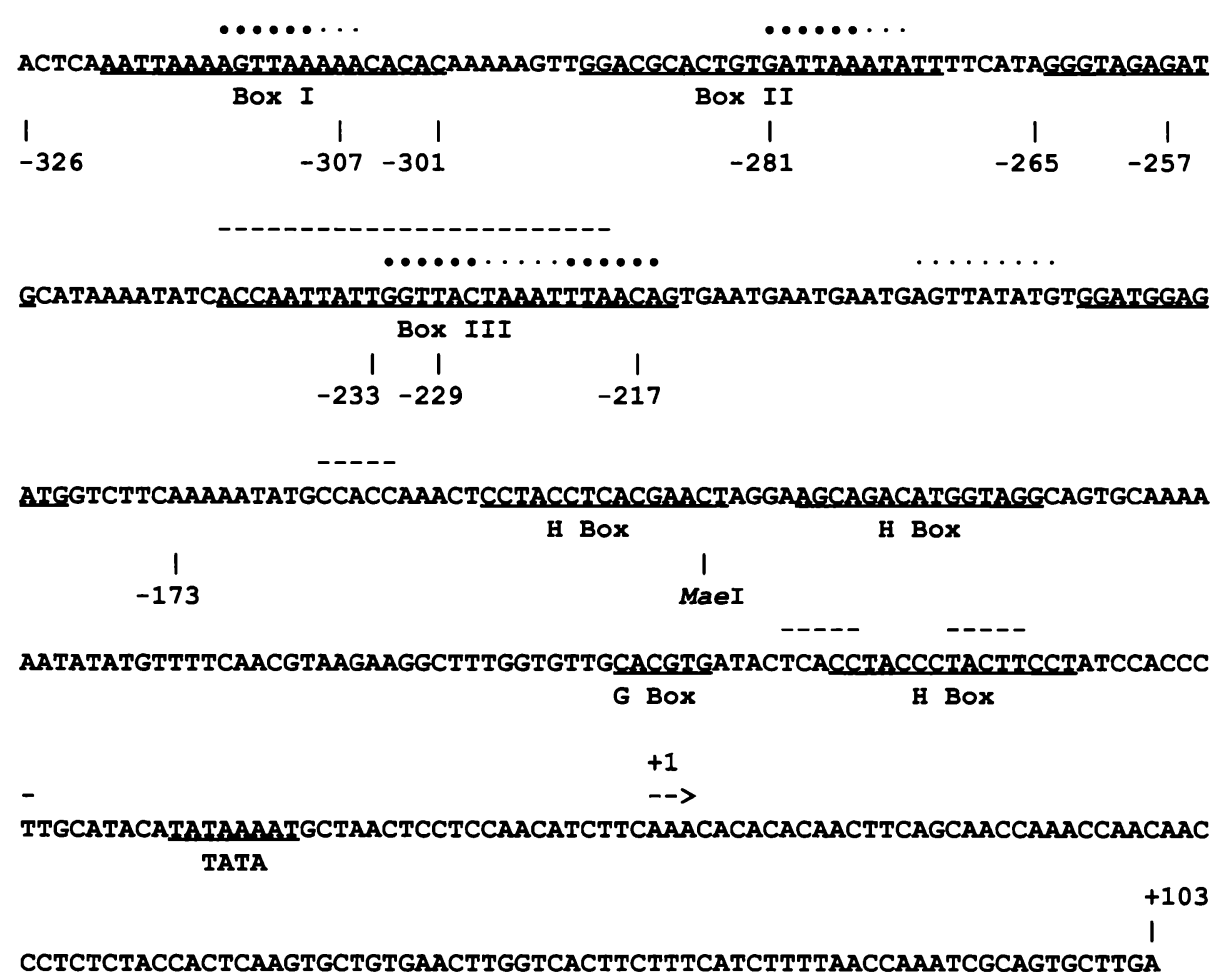

Fig. 1. Sequence and related features of the bean $C H S 15$ promoter. DNase I-footprinted regions using bean suspension cell nuclear extracts (SBF-1) are underlined and labelled Box I, Box II, and Box III. The positions of the 6 bp sequences (large dots) with consensus homology to the GT-1 core binding site GGTTAA, and 3 bp extended regions of consensus homology $\left({ }^{A} / T^{A} / T^{A} / T\right.$, small dots) are indicated above the sequence. Also indicated with small dots is a sequence with lesser homology to these core and extended sequences. The three $\mathrm{H}$ boxes and the $\mathrm{G}$ box are underlined and labelled $\mathrm{H}$ Box and $\mathrm{G}$ Box. Also underlined are two sequence elements with the consensus GGPuTPuGAGATG and the TATA box. Additional regions with sequence similarity to regions of the bean PAL2 promoter are indicated with a dashed overline. Two of these regions show sequence similarity to the $P A L 2$ promoter element CCACCAACCCC [18], while the third region overlaps SBF-1 box III. The deletion endpoints of the CHS 15 promoter-deletion constructs examined in this study are also shown.

not possible to analyze the developmental patterns of CHS15 expression in bean by direct methods such as in situ hybridization. In addition, stable transformation and regeneration of bean cannot yet be readily achieved, thus analysis of the CHS promoter reporter gene fusions are also not possible in the homologous system. Therefore in order to analyze the patterns of CHS15 gene expression in planta and in particular to target the distal region of the promoter, and by implication SBF-1, we generated transgenic tobacco plants in which the activity of an introduced GUS reporter gene is driven by a nested promoter series sequentially removing box I, II, and III. We show activation of expression in ini- tiating tobacco lateral roots and in developing seeds and demonstrate that specific deletions within the box I, II, III region modulate expression. We further show that specific DNA-binding activities consistent with the results obtained, are present in bean nuclear extracts.

\section{Materials and methods}

\section{Plasmid constructions}

Plasmids pBICHS1 and pBICHS2 were created by ligating the $\mathrm{CHS} 15$ promoters $\mathrm{pCHC1}(-326$ to +103 relative to the transcriptional start site) 
and $\mathrm{pCHC} 2(-173$ to +103 relative to the transcriptional start site), described by Dron et al. [5], into the binary vector pBI101.1 [14, 15]. The 5 -deleted promoter constructs were created by cleaving a plasmid containing the CHS15 promoter from positions -326 to +103 , with $S p h$ I and $S a l$ I, followed by treatment with exonuclease III and mung bean nuclease. Deleted promoter fragments were repaired with the Klenow fragment of DNA polymerase, recloned into pUC19, and the deletion endpoints were determined by dideoxy chain-termination sequencing [27]. Deleted promoter fragments were released from the plasmids with either Hind III and Bam HI or $X b a \mathrm{I}$, gel-purified, and ligated into the binary vector $\mathrm{pBI} 101.1[14,15]$. All plasmid constructions examined in this work are listed in Table 1.

\section{Plant materials and transformation}

The vector plasmids carrying the CHS15 promoter-GUS constructs were transferred from $E s$ cherichia coli into Agrobacterium tumefaciens strain LBA4404 by direct DNA transfer [1]. Leaf disk transformation of Nicotiana tabacum cv. Xanthi and regeneration of transgenic plants was performed as described [24].

Total DNA was prepared from leaf tissue of kanamycin resistant plants by the Dellaporta method as described by Herrera-Estrella and Simpson [12], and Southern border analysis was used to show integration of the gene constructs into the tobacco genome. DNA was digested with Eco RI or Hind III, separated by gel electrophoresis, blotted and probed with GUS or NPTII probes using standard methods [26]. Independent integration of T-DNA into the tobacco genome was demonstrated for each tobacco line examined in this study.

\section{GUS assays}

Histochemical localization of GUS activity in transgenic tobacco seedlings was performed as previously described [14] with the addition of
Table 1. Summary of the independent transgenic tobacco lines examined in this study, together with the patterns of histochemical GUS localization observed in transgenic tobacco seeds and initiating lateral roots.

\begin{tabular}{|c|c|c|c|c|}
\hline \multirow{2}{*}{$\begin{array}{l}\text { Promoter } \\
\text { deletion }\end{array}$} & \multirow{2}{*}{$\begin{array}{l}\text { Construct } \\
\text { designation }\end{array}$} & \multirow{2}{*}{$\begin{array}{l}\text { Independent } \\
\text { transgenic } \\
\text { lines }\end{array}$} & \multicolumn{2}{|c|}{ GUS activity in } \\
\hline & & & seeds & $\begin{array}{l}\text { initiating } \\
\text { lateral } \\
\text { roots }\end{array}$ \\
\hline \multicolumn{5}{|l|}{ Undeleted } \\
\hline-326 & $\mathrm{pBICHCl}$ & $\begin{array}{l}\text { CHS } 1.1 \\
\text { CHS } 1.8 \\
\text { CHS } 1.9\end{array}$ & $\begin{array}{l}+ \\
+ \\
+\end{array}$ & $\begin{array}{l}+ \\
+ \\
+\end{array}$ \\
\hline \multicolumn{5}{|l|}{ Box I } \\
\hline$\Delta-307$ & pBICHS3.5.1 & $\begin{array}{l}\text { CHS 3.5.1.1 } \\
\text { CHS3.5.1.2 } \\
\text { CHS3.5.1.3 } \\
\text { CHS3.5.1.4 } \\
\text { CHS3.5.1.7 }\end{array}$ & $\begin{array}{l}+ \\
+ \\
+ \\
- \\
+\end{array}$ & $\begin{array}{l}+ \\
+ \\
+ \\
- \\
+\end{array}$ \\
\hline$\Delta-301$ & pBICHS3.1.1 & $\begin{array}{l}\text { CHS3.1.1.1 } \\
\text { CHS3.1.1.2 } \\
\text { CHS3.1.1.3 } \\
\text { CHS3.1.1.4 } \\
\text { CHS3.1.1.6 }\end{array}$ & $\begin{array}{l}+ \\
+ \\
+ \\
+ \\
+\end{array}$ & $\begin{array}{l}+ \\
+ \\
+ \\
+ \\
+\end{array}$ \\
\hline $\begin{array}{l}\text { Box I }+ \text { E } \\
\Delta-281\end{array}$ & $\begin{array}{l}\text { 3ox II } \\
\text { pBICHS3.7.5 }\end{array}$ & $\begin{array}{l}\text { CHS3.7.5.1 } \\
\text { CHS3.7.5.2 }\end{array}$ & $\begin{array}{l}+ \\
-\end{array}$ & $\begin{array}{l}- \\
-\end{array}$ \\
\hline$\Delta-265$ & pBICHS3.7.2 & $\begin{array}{l}\text { CHS3.7.2.1 } \\
\text { CHS3.7.2.3 }\end{array}$ & $\begin{array}{l}+ \\
+\end{array}$ & - \\
\hline$\Delta-257$ & pBICHS3.10.4 & $\begin{array}{l}\text { CHS3.10.4.1 } \\
\text { CHS3.10.4.3 } \\
\text { CHS3.10.4.4 }\end{array}$ & $\begin{array}{l}- \\
- \\
+\end{array}$ & $\begin{array}{l}- \\
- \\
-\end{array}$ \\
\hline $\begin{array}{l}\text { Box I }+ \text { E } \\
\Delta-233\end{array}$ & $\begin{array}{l}\text { 3ox II + Box III } \\
\text { pBICHS3.5.3 }\end{array}$ & $\begin{array}{l}\text { CHS3.5.3.1 } \\
\text { CHS3.5.3.4 } \\
\text { CHS3.5.3.5 }\end{array}$ & $\begin{array}{l}+ \\
+ \\
-\end{array}$ & $\begin{array}{l}- \\
- \\
-\end{array}$ \\
\hline$\Delta-229$ & pBICHS 1.2 & $\begin{array}{l}\text { CHS } 1.2 .1 \\
\text { CHS } 1.2 .3\end{array}$ & $\begin{array}{l}+ \\
+\end{array}$ & - \\
\hline$\Delta-217$ & pBICHS5.3 & $\begin{array}{l}\text { CHS5.3.1 } \\
\text { CHS5.3.2 } \\
\text { CHS5.3.3 }\end{array}$ & $\begin{array}{l}+ \\
+ \\
+\end{array}$ & $\begin{array}{l}- \\
- \\
-\end{array}$ \\
\hline Boxes I-II & I and $-217 /-17$ & 3 region & & \\
\hline$\Delta-173$ & pBICHC2 & $\begin{array}{l}\text { CHS2.1 } \\
\text { CHS2.3 } \\
\text { CHS2.5 } \\
\text { CHS2.8 } \\
\text { CHS2.9 }\end{array}$ & $\begin{array}{l}- \\
- \\
+ \\
+ \\
-\end{array}$ & $\begin{array}{l}- \\
- \\
+ \\
+ \\
-\end{array}$ \\
\hline
\end{tabular}

Histochemically detectable GUS activity observed (+), or not observed ( - ). 
$0.1 \%$ Triton $\mathrm{X}-100$ as a wetting agent. After incubation at $37^{\circ} \mathrm{C}$ for $16 \mathrm{~h}$, plant tissues were fixed in $5 \%$ formaldehyde, $5 \%$ acetic acid, $20 \%$ ethanol for $2 \mathrm{~h}$, then cleared in $50 \%(\mathrm{v} / \mathrm{v})$ ethanol for $2 \mathrm{~h}$ followed by $95 \%$ ethanol overnight. Alternatively, after incubation, plants were placed in $50 \%(\mathrm{v} / \mathrm{v})$ ethanol for $2 \mathrm{~h}$, then $95 \%$ ethanol until tissues had cleared, and photographed immediately thereafter. Plantlets from several lines were also stained according to the following protocol: each seedling was rinsed once in $50 \mathrm{mM}$ sodium phosphate buffer ( $\mathrm{pH} 7.0)$ containing $0.1 \%(\mathrm{v} / \mathrm{v})$ Triton X-100, $1.0 \mathrm{mM}$ EDTA, and $10 \%(\mathrm{v} / \mathrm{v})$ methanol. The seedlings were then transferred to the GUS reaction buffer which was identical to the rinse buffer except that it also contained $0.5 \mathrm{mM}$ potassium ferricyanide $/ 0.5 \mathrm{mM}$ potassium ferrocyanide and $0.5 \mathrm{mg} / \mathrm{ml}$ 5-bromo-4chloro-3 indolylglucuronide (X-gluc). No difference in staining pattern was observed between tissue stained with or without the presence of potassium ferricyanide/potassium ferrocyanide. Histochemical localization of GUS activity in developing seeds was performed on sectioned seed pods as described [14] with the addition of $0.1 \%$ Triton X-100. After overnight incubation at $37{ }^{\circ} \mathrm{C}$ the seed pods were bleached in $0.5 \%$ hypochlorite for up to $10 \mathrm{~min}$.

\section{Preparation of nuclear extracts for gel retardation analysis}

Nuclear extracts were prepared by a modification of the method of Staiger et al. [29]. A $33 \mathrm{~g}$ portion of frozen tissue (suspension cells, roots, or seeds) was ground to a fine powder under liquid nitrogen, suspended in $3 \mathrm{vol}(\mathrm{w} / \mathrm{v})$ of ice-cold nuclear isolation buffer (NIB) $(25 \mathrm{mM}$ MES/ $\mathrm{KOH}, \mathrm{pH}$ 6.0, $5 \mathrm{mM}$ NaEDTA pH 8.0, $10 \mathrm{mM}$ $\mathrm{KCl}, 250 \mathrm{mM}$ sucrose, $0.5 \mathrm{mM}$ spermine, $0.5 \mathrm{mM}$ spermidine phosphate, $1.0 \mathrm{mM}$ DTT, $0.5 \% \mathrm{v} / \mathrm{v}$ Triton X-100, 1.0 mM phenylmethylsulfonylfluoride (PMSF), and $1 \mathrm{mg} / \mathrm{ml}$ each of leupeptin, pepstatin A, chymostatin, and antipain), and homogenized twice for $30 \mathrm{~s}$ with a Brinkmann polytron at setting 3.0-3.5. All subsequent operations were performed at $4{ }^{\circ} \mathrm{C}$. The slurry was filtered through one layer of miracloth, then through one layer of $30 \mathrm{~mm}$ or $40 \mathrm{~mm}$ nylon mesh. The resulting solution was centrifuged at $2000 \times g$ for $10 \mathrm{~min}$. The pellet containing nuclei and additional cellular debris was resuspended in $10 \mathrm{ml}$ Percoll float buffer $(3 \mathrm{ml} 5 \times \mathrm{NIB}+22.5 \mathrm{~g}$ Percoll) and centrifuged at $4500 \times g$ for $5 \mathrm{~min}$. The surface paste of nuclei was collected, resuspended in $10 \mathrm{ml}$ Percoll float buffer and recentrifuged at $4500 \times \mathrm{g}$ for $5 \mathrm{~min}$. The float procedure was repeated an additional two times for seed nuclear extracts. The final surface paste of nuclei was retained and washed twice in NIB minus Triton $\mathrm{X}-100$ by centrifugation at $2000 \times \mathrm{g}$ for $10 \mathrm{~min}$. The resulting nuclear pellet was resuspended in approximately 3 volumes nuclear extraction buffer (20 mM HEPES/KOH pH 7.9, $1.5 \mathrm{mM}$ $\mathrm{MgCl}_{2}, 25 \% \mathrm{v} / \mathrm{v}$ glycerol, $0.2 \mathrm{mM}$ NaEDTA PH $0.8,420 \mathrm{mM} \mathrm{NaCl}, 1.0 \mathrm{mM}$ DTT, $0.5 \mathrm{mM}$ PMSF, and $0.5 \mathrm{mg} / \mathrm{ml}$ each of leupeptin, pepstatin A, chymostatin, and antipain), incubated on ice for $30 \mathrm{~min}$ with regular mixing, then centrifuged at $25000 \times g$ for $30 \mathrm{~min}$. The supernatant containing nuclear proteins was dialyzed against $1000 \mathrm{ml}$ of dialysis buffer $(20 \mathrm{mM}$ HEPES $/ \mathrm{KOH}$ $\mathrm{pH} 7.9,20 \% \mathrm{v} / \mathrm{v}$ glycerol, $50 \mathrm{mM} \mathrm{KCl}, 0.1 \mathrm{mM}$ NaEDTA pH 8.0, 1.0 mM PMSF, and $1.0 \mathrm{mM}$ DTT) over $1.5 \mathrm{~h}$ using a Pierce microdialysis unit and membrane (molecular weight cut-off 8000) and five $180 \mathrm{ml}$ chamber flushes. This ensures that more than $95 \%$ of the salt has been removed. The dialyzed extracts were centrifuged at maximum speed in a microcentrifuge (Sorval Microspin 245) for $15 \mathrm{~min}$ to remove any precipitate prior to being snap frozen in $50 \mathrm{ml}$ aliquots in liquid nitrogen, and stored at $-70{ }^{\circ} \mathrm{C}$. Protein concentrations were determined against BSA standards by the Bradford microassay (BioRad) according to the manufacturer's instructions.

\section{Assay for binding activity}

Binding activity in nuclear extracts was monitored by gel retardation analysis (GRA) in $10 \mu \mathrm{l}$ reaction volumes. Reaction mixes contained ei- 


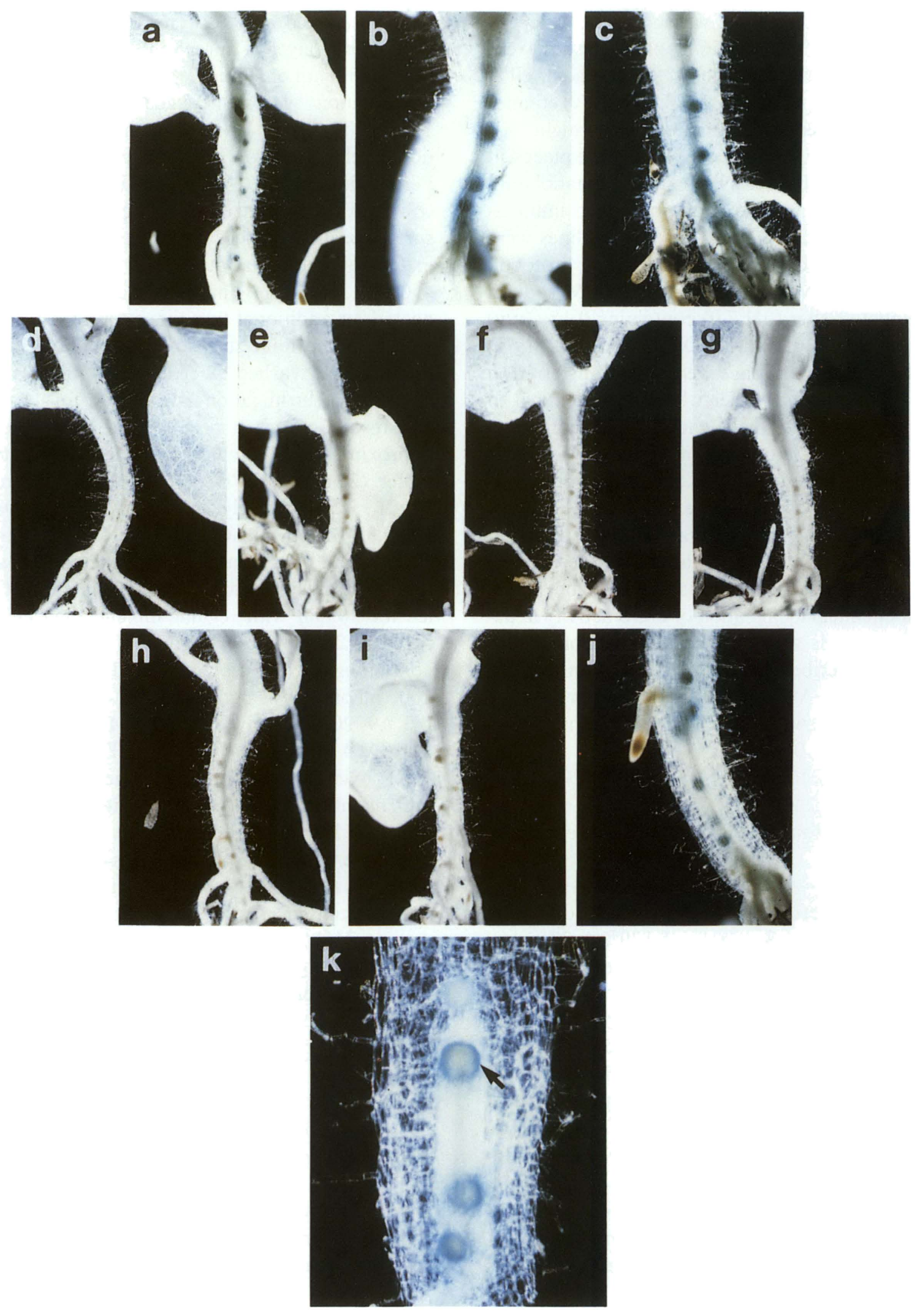


ther a cloned ${ }^{32} \mathrm{P}$-labelled, unidirectional, trimeric concatamer of box III ('box III trimer'; the unligated box III sequence is given in Harrison et al. [10]), a cloned ${ }^{32}$ P-labelled Hind III-Mae I fragment of the CHS15 promoter (Mae I-1 [11]), or a cloned ${ }^{32} \mathrm{P}$-labelled, unidirectional, tetrameric concatamer of the oligonucleotides 5'-AAAGAATGAATGAATGAGTTATATGTGGATGGAGATGGTCTT-3' and 5' -TTTAAGACCATCTCCATCCACATATAACTCATTCATTCATTC-3' which span positions -213 to -175 (inclusive) of the CHS15 promoter (henceforth ' $-213 /-175$ fragment' or ' $-213 /-175$ tetramer'). The box III trimer cloned in pGEM7 was released by digestion with Eco RI and $S a l$ I. The Mae I-1 fragment cloned in pIBI24 was released by digestion with Eco RI and Bam HI. The $-213 /-175$ fragment tetramer cloned in pBluescript II was released by digestion with Eco RI and Hind III. The appropriate fragments were gel-purified and labelled by end filling with $\left[\alpha-{ }^{32} \mathrm{P}\right] \mathrm{dATP}[26]$. Binding reactions were performed in a buffer containing $10 \mathrm{mM}$ Tris- $\mathrm{HCl}$ $\mathrm{pH} 7.5,50 \mathrm{mM} \mathrm{NaCl}, 1.0 \mathrm{mM}$ dithiothreitol, $0.5 \mathrm{mM}$ PMSF, $1.0 \mathrm{mM}$ EDTA pH 8.0, and $4 \%$ $(\mathrm{v} / \mathrm{v})$ glycerol. The concentration of poly(dI$\mathrm{dC}) \cdot(\mathrm{dI}-\mathrm{dC})$ used in each reaction mix was determined by titration but was $100 \mathrm{ng} / \mu \mathrm{l}$ for all results shown here. Binding reactions contained $0.1 \mathrm{ng}$ labelled probe (generally $50 \mathrm{cps}$ ) and were initiated by addition of labelled probe immediately (1 min) after addition of test extract, followed by incubation on ice for $30 \mathrm{~min}$. The amount of protein extract added to each binding reaction is indicated in the figure legends. Samples were analyzed by electrophoresis through a $4 \%$ polyacrylamide $(60: 1)$ gel in $0.25 \times \mathrm{TBE}$ at $4{ }^{\circ} \mathrm{C}$. Gels were dried under vacuum and exposed for $2-12 \mathrm{~h}$ at $-70{ }^{\circ} \mathrm{C}$ to Kodak X-Omat X-ray film. Competition GRAs were performed as described above, but included competitor DNA oligonucle- otides at the molar excess indicated in the figures. Competition GRA's for SBF-1 activity utilized a cloned unidirectional, tetrameric concatamer of a box III 'double mutant' [10] that was released from pGEM7 by Eco RI/Bam HI digestion. The box III 'double mutant' carries a one nucleotide mutation in each of its two SBF-1 core recognition sites compared with box III (5'-GGTTAC-3' becomes $5^{\prime}$-GCTTAC-3' and, on the opposite strand, 5' -TGTTAA-3' becomes 5'-TCETAA$\left.3^{\prime}\right)$.

\section{Results}

Transformation of tobacco with nested CHS 15 promoter: GUS constructs

A fragment of the $\mathrm{CHS} 15$ promoter from positions -326 to +103 relative to the transcription start site, together with a series of $5^{\prime}$ deletions which sequentially remove boxes I, II, and III (Table 1 and Fig. 1) were fused to the promoterless GUS-coding sequence in the binary vector pBI101.1. These constructs were used to transform tobacco and transgenic plants were regenerated under kanamycin selection.

Independent integration of T-DNA into the tobacco genome was demonstrated for all the tobacco lines described here (data not shown). Copy number varied between 1 to 4 inserts depending on plant line, but was not correlated to the all or nothing expression patterns described below.

Activation of the CHS15 promoter in initiating lateral roots

Histochemical staining of whole tobacco seedlings for GUS activity revealed that the -326

Fig. 2. Activation of the CHS15 promoter in initiating lateral roots determined by histochemical localization of GUS activity in situ. a to $\mathrm{j}$ show the presence or absence of histochemically detectable GUS activity in initiating lateral roots of whole stained plants, while k shows a section through stained initiating lateral roots, showing staining in peripheral lateral root cells (arrowed). a, -326 , b, $\Delta-307$; c, $\Delta-301$; d, $\Delta-281$; e, $\Delta-265$; f, $\Delta-257$; g, $\Delta-233$; h, $\Delta-229$; i, $\Delta-217$; j, $\Delta-173$; k, $\Delta-307$. Magnification of figures: a, d, e, $11 \times$; b, $21 \times$; c, $14 \times ;$ j, $18 \times ; \mathrm{k}, 28.5 \times$. 

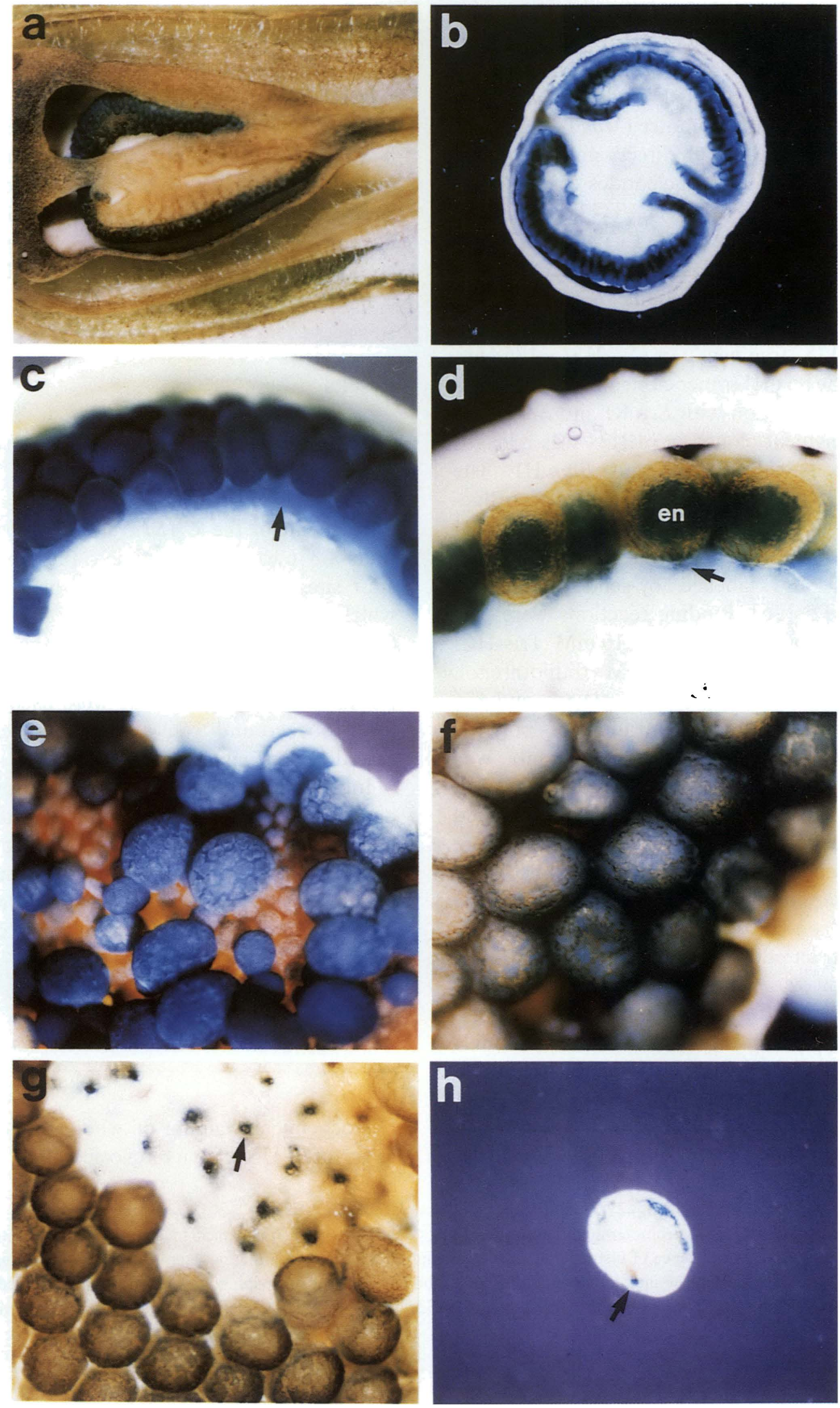
CHS15 promoter (Fig. 2a), the box I-deleted promoter constructs $\Delta-307$ (Fig. 2b), $\Delta-301$ (Fig. 2c), and the $\Delta-173$ construct (Fig. 2 j), were activated in initiating lateral roots. These CHS 15 promoters were activated within the pericycle of the primary root early in lateral root development, and activation persisted until emergence of the lateral roots from the primary root. GUS staining was not observed following emergence of the lateral root from the primary root (Fig. 2j). GUS activity was primarily directed to peripheral cell layers in the emerging lateral root (Fig. 2k).

In contrast to promoter:GUS constructs that deleted box I ( $\Delta-307$ and $\Delta-301$, Figs. $2 \mathrm{~b}, \mathrm{c})$, lines carrying constructs that deleted box II $(\Delta-281$, $\Delta-265$, and $\Delta-257$ ) showed no histochemically detectable GUS activity in initiating lateral roots (Figs. 2d, e, f). Tobacco lines carrying constructs that deleted box III $(\Delta-233, \Delta-229$, and $\Delta-217)$ similarly showed no histochemically detectable GUS activity in initiating lateral roots (Figs. $2 \mathrm{~g}$, h, i). In contrast, deletion of an additional $43 \mathrm{bp}$ to position -173 , including $19 \mathrm{bp} 5^{\prime}$ of the first H-box, reactivated GUS activity in initiating lateral roots (Fig. 2j). Results are summarized in Table 1.

These results suggest that qualitative expression in initiating lateral roots is activated by sequence elements located downstream from position -173 . Our data further suggest that this downstream-element-directed activation of expression is modulated both by SBF-1 box II, which acts as a transcriptional enhancer or coactivator, and by a silencer element that is deleted or disrupted in lines carrying $\Delta-173$ constructs (Fig. j), but not in lines carrying box III-deleted $(\Delta-233, \Delta-229, \Delta-217$, [Figs. $2 \mathrm{~g}, \mathrm{~h}, \mathrm{i}])$ constructs.
Activation of the CHS15 promoter in developing seeds

Histochemical staining of longitudinally and cross-sectioned seed pods from mature tobacco plants revealed that lines carrying -326 CHS15 promoter-GUS constructs express GUS activity in developing seeds, primarily in the endosperm and in epidermal cells. GUS activity was observed in all tissues of the seed during the initial stages of seed development (1-4 days after anthesis) but was not observed in any additional maternal tissues in the developing seed pod (Figs. 3a, b and c), except for the placenta. GUS activity was confined to the endosperm surrounding the globular stage embryo by 4-6 days after anthesis (Fig. 3d). During this time, the cell layers exterior to the endosperm, including the seed integuments and the epidermis were unstained. From 5-10 days after anthesis, as the seed attains maximum size and embryo development begins to proceed beyond the globular stage, GUS activity was redirected primarily to the crenulated epidermal cells of the seed coat (Fig. $3 e$ and $f$ ) and to the placenta at the point of seed attachment (Fig. 3g). Expression in the placenta was contiguous with expression in the funiculus (Fig. 3h). GUS activity declined prior to the accumulation of anthocyanins and other pigments in the seed coat, although it occasionally persisted in the placenta for several additional days.

Localization of GUS activity to developing seeds was observed for all promoter deletions examined, including deletion to position -173 (Table 1). Hence, these results involving seed expression, like the results for initiating lateral root expression, suggest that qualitative expression in

Fig. 3. Activation of the CHS15 promoter in developing seeds determined by histochemical localization of GUS activity in situ. All figures show the histochemical localization of GUS activity directed by the -326 promoter. a, b, c, CHS 15 promoter activation throughout seeds and in the placenta (arrowed in c) 1-4 days after anthesis; d, activation of the CHS15 promoter in the endosperm (en) and the placenta (arrowed) 4-6 days after anthesis; e, f, activation of the CHS15 promoter in seed epidermal cells 5-10 days after anthesis viewed primarily under incident (e) or transmitted (f) light; g, activation of the CHS15 promoter in the placenta (arrowed) at, and immediately surrounding, the point of attachment of the funiculus; h, activation of the CHS15 promoter in the funiculus (arrowed). Magnification of figures: a, $11 \times ; \mathrm{b}, 7.3 \times ; \mathrm{c}, \mathrm{d}, \mathrm{e}, \mathrm{f}, \mathrm{h}, 28 \times ; \mathrm{g}, 22 \times$. 


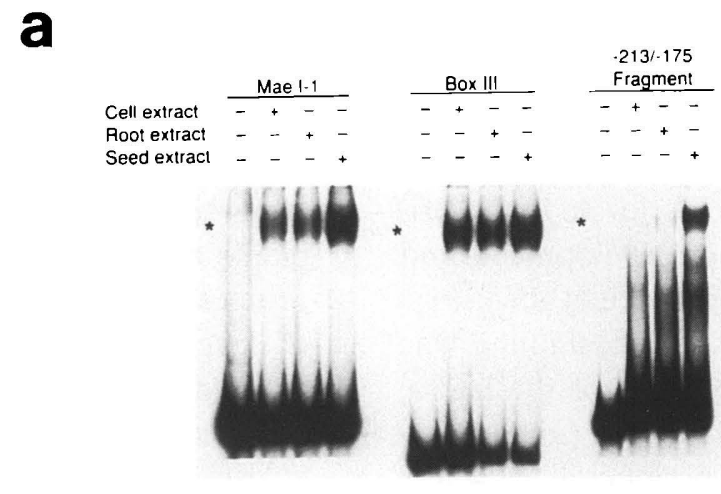

b

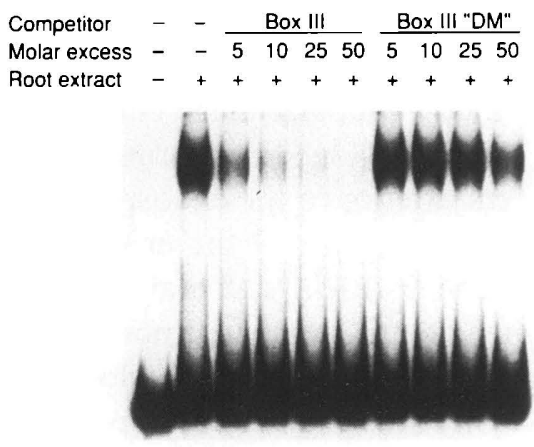

C Competitor
Molar excess Seed extract -+++++++++
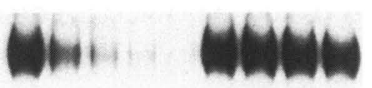

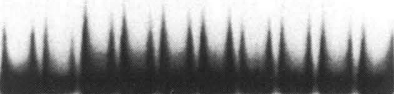

e

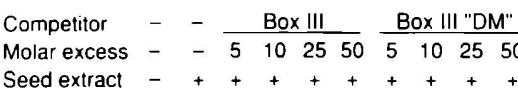
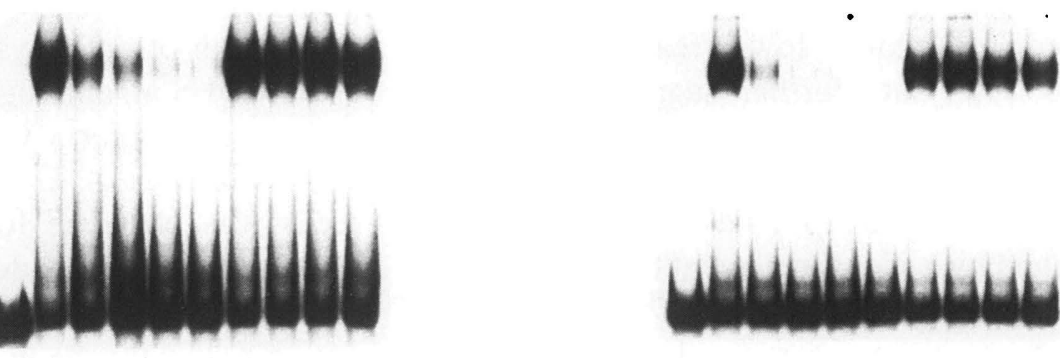

f

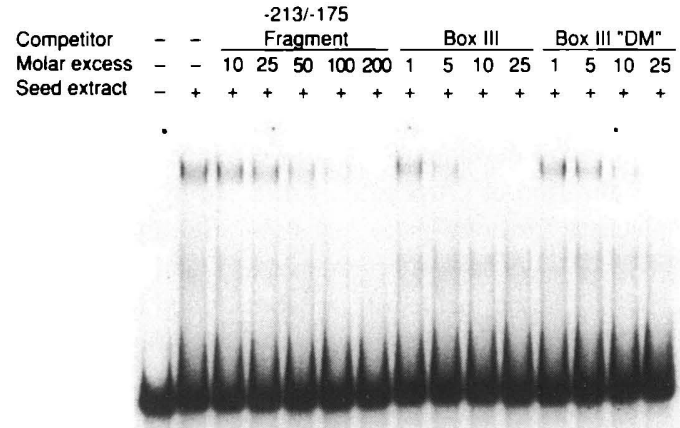


developing seeds is activated by sequence elements located downstream from position -173 .

CHS expression has been previously reported in other plant tissues including the pigmented part of the corolla and in wounded leaves [28]. However, CHS15-GUS constructs did not express histochemically detectable GUS activity in these tissues.

\section{DNA-binding activities in bean nuclear extracts}

If the promoter-deletion studies in tobacco correctly report the regulation of CHS 15 transcription in bean, then one would predict the presence, in nuclear extracts of bean roots, of SBF-1 (or a related) activity, and $-217 /-173$ region-binding activity. Thus, we used gel retardation analysis (GRA) to detect the presence of trans-acting factors in bean roots and seeds that are able to bind to one or more of the Mae I-1 fragment of the CHS15 promoter, a SBF-1 box III trimer, or a $-213 /-175$ tetramer. The Mae I-1 fragment of the CHS15 promoter contains three DNase-Ifootprinted regions that each bear either one or two elements with a consensus SBF-1 binding motif GGTTAA(A/T)(A/T)(A/T). SBF-1 box III, the third DNase-I-footprinted region present in Mae I-1, contains two SBF-1 consensus binding motifs on opposite DNA strands. SBF-1 box III was used as a probe rather than SBF-1 box II, since previous results have shown that SBF-1 has higher affinity for box III than for box II [ 10 , 17]. The $-213 /-175$ fragment excludes sequences overlapping positions -217 and -173 that are footprinted with bean and/or alfalfa nuclear extracts $[10,17]$.

Mae I-l fragment- and box III trimer-binding activities were observed in nuclear extracts of bean roots and seeds (Fig. 4a). SBF-1 activity was identified in bean suspension cells in control experiments using the same method to isolate nuclear proteins (Fig. 4a). The bean root, seed, and cell culture Mae I-1 fragment- and box IIIbinding activities retarded the probes to the same positions (Fig. 4a). An activity was also present in nuclear extracts of bean roots and seeds that binds to the $-213 /-175$ fragment tetramer (Fig. 4a). This activity was not detected in bean suspension cell nuclear extracts (Fig. 4a).

The Mae I-1 fragment- and box III trimerbinding activities present in bean roots and seeds were maintained in the presence of $1000 \mathrm{ng}$ (10000-fold weight excess) sheared poly(dI$\mathrm{dC}) \cdot(\mathrm{dI}-\mathrm{dC})$ as non-specific competitor DNA. This molar excess of poly $(\mathrm{dI}-\mathrm{dC}) \cdot(\mathrm{dI}-\mathrm{dC})$ was used in all experiments shown in the Fig. 4a-e. Competition GRA using either the Mae I-1 fragment (Fig. 4b, c) or the box III trimer (Fig. 4d, e) as ${ }^{32} \mathrm{P}$-labelled probes demonstrated that these binding activities are sequence specific. Thus, the activities binding to the Mae I-1 fragment and to the box III trimer were competed by excess unlabelled box III trimer but only very weakly competed by an equimolar excess of a box III 'double mutant' tetramer (Fig. 4b-e). Hence, the Mae I-1 fragment and box III-binding activities present in bean root and bean seed nuclear extracts are indistinguishable, in terms of their binding characteristics, from SBF-1 activity present in bean suspension cell nuclear extracts. Additional weak complexes with faster mobilities than the SBF-1/ DNA complex were variably observed, but were not differentially competed, and therefore do not represent sequence-specific binding activities.

A $-213 /-175$ fragment-binding activity was

Fig. 4. CHS15 promoter-binding activities present in bean nuclear extracts. a shows Mae I-1 fragment-, SBF-1 box III trimer-, and $-213 /-175$ fragment tetramer-binding activities present in nuclear extracts of bean suspension cells, roots, and seeds. The Mae I-1 and box III assays with cell and root extracts contain $1.2 \mu \mathrm{g}$ of total protein. The assays with seed extracts contain $0.16 \mu \mathrm{g}$ of total protein. Assays with the $-213 /-175$ fragment each contain $1.2 \mu \mathrm{g}$ of total protein from either cell root or seed. Retarded protein-DNA complexes are indicated with an asterix. $b$ to e show competition gel retardation analysis of the binding of bean root (b, d) or bean seed (c, e, f) nuclear protein(s) to either the Mae I-1 fragment of the CHS15 promoter (b, c), the SBF-1 box III trimer $(\mathrm{d}, \mathrm{e})$, or the $-213 /-175$ fragment tetramer (f). Competition assays with roots extracts contain $1.2 \mu \mathrm{g}$ of protein. Competition assays with seed extracts contain $0.16 \mu \mathrm{g}$ of protein. Unlabelled competitor DNAs (box III trimer, box III 'double mutant' tetramer, or $-216 /-174$ fragment tetramer) were included in incubations at the indicated molar excess. 
present in nuclear extracts of bean roots and seeds (Fig. 4a) but not in extracts prepared from cell culture. The activity was weak in root extracts; however, it was reproducible. In both extracts the activity was maintained in the presence of at least $1000 \mathrm{ng}$ (10000-fold weight excess) sheared poly $(\mathrm{dI}-\mathrm{dC}) \cdot(\mathrm{dI}-\mathrm{dC})$ as non-specific competitor DNA and this excess of poly $(\mathrm{dI}-\mathrm{dC}) \cdot(\mathrm{dI}-\mathrm{dC})$ was used in the experiment shown in Fig. 4f. Competition GRA with the $-213 /-175$ tetramer as ${ }^{32} \mathrm{P}$ labelled probe demonstrated that the binding activity present in bean seed nuclear extracts is sequence-specific (Fig. 4f). Thus, the $-213 /-175$ tetramer-binding activity was completed by excess unlabelled $-213 /-175$ tetramer. Interestingly, the $-213 /-175$ tetramer-binding activity was strongly competed by the box III trimer, and less strongly competed by the box III 'double mutant' tetramer suggesting that this binding activity recognizes part of the box III sequence but that the $G$ residues are not critical for binding. Both these oligonucletides showed much greater affinity for $-213 /-175$ tetramer-binding activity than did the $-213 /-175$ tetramer itself (Fig. 4f). Very similar results were also obtained for the comparatively low abundance $-213 /-175$ tetramer-binding activity present in bean root nuclear extracts (data not shown).

\section{Discussion}

Our data show that the CHS15 promoter is active in developing seed and during lateral root initiation. In addition, fine qualitative control of CHS 15 promoter activation in initiating tobacco lateral roots is modulated by specific sequence elements located between positions -173 and -326 . Hence, deletion of SBF-1 box II eliminated expression of GUS reporter gene activity in initiating lateral roots, but failed to eliminate expression in developing seeds. This suggests that SBF-1 box II functions as a qualitative coactivator or enhancer of expression in initiating lateral roots. Alternatively, the requirement for box II could support a model whereby at least two SBF-1 boxes in close proximity to each other are required for efficient binding of the requisite tobacco or bean (SBF-1) factor(s). Lawton et al. [17] have shown that this is the case for SBF-1 activity present in bean suspension cell nuclear extracts.

Deletion of box III failed to restore GUS activity to initiating lateral roots, but further deletion of an additional $43 \mathrm{bp}$ to position -173 , $19 \mathrm{bp} 5^{\prime}$ to the first $\mathrm{H}$ box, reestablished expression. This suggests that a silencer element is located between SBF-1 box III and position -173 that is deleted or internally disrupted by the -173 deletion. Studies of nested CHS15 gene promoterCAT fusions in electroporated alfalfa protoplasts have similarly shown that the -217 (box III) deleted promoter directs lower CAT reporter activity that the -173 deleted promoter [10].

While qualitative control of $\mathrm{CHS} 15$ promoter activation in initiating tobacco lateral roots is modulated by specific sequence elements located between positions -326 and -173 , our data show that activation of expression is mediated by sequence elements located downstream from position -173 . Activation of expression in developing seeds is similarly controlled by sequence elements located downstream from position -173 . Candidate downstream sequence elements include the G- and/or H-boxes that are involved in transcriptional activation of CAT reporter gene activity in electroporated protoplasts in the presence of trans-p-coumaric acid [20,21]. Thus, $\mathrm{H}-$, and/or G-box activators may interact with upstream modulators, including at least one SBF-1 box, to direct transcription in lateral roots. Similar interactions between G-box, GT-1 box (GGTTAA), and I-box (GATAAG) motifs are thought to play a role in coordinating light-regulated expression of the $r b c s 3 A$ gene [4].

Extrapolation of the expression patterns observed in transgenic tobacco to bean predicts that trans-acting factors should be present in nuclear extracts of bean roots that bind to Mae I-1 fragment, SBF-1 box III, and $-213 /-175$ fragment probes. Consistent with the tobacco data, we have identified each of these predicted activities in bean root nuclear extracts. In addition, we also identified the presence of Mae I-1 fragment, SBF-1 
box III, and $-213 /-175$ fragment binding activities in bean seed nuclear extracts. The tobacco data predict that the $-326 /-173$ region encompassing each of the three probes is not involved in modulating CHS15 promoter activity in developing seeds. However, bean seeds yielded at least a ten-fold higher ratio of binding activity:total extracted protein than either bean roots or bean suspension cells (G. Hotter, unpublished results). It is possible that SBF-1 activity in bean seeds may not modulate CHS 15 activation in seeds, but may instead modulate activation of other genes with SBF-1-binding sites in their promoters.

We have shown that unlabelled box III trimer strongly competes the Mae I-1 fragment- and SBF-1 box III trimer-binding activities present in both bean root and seed nuclear extracts, while a mutated version of box III competes only very weakly. Very similar results have been reported for SBF-1 activity present in both bean suspension cell nuclear extracts and alfalfa suspension cell nuclear extracts [10, 17]. Thus, the Mae I-1 fragment- and box III-binding activities present in nuclear extracts of bean roots are indistinguishable in their DNA-binding characteristics from SBF-1. We suggest that these activities either represent SBF-1, or one or more closely related proteins.

Our results suggest that a silencer of expression in initiating lateral roots is located between SBF-1 box III and position -173 and that this element is deleted or internally disrupted by the -173 deletion. In vitro DNase I footprinting of the CHS15 promoter using alfalfa nuclear extracts has identified a footprinted region overlapping position -173 at its $5^{\prime}$ end (Fig. 1) [10]. However, this region is not footprinted in equivalent experiments using bean nuclear extracts [17], suggesting that an equivalent activity is not present in bean. Our data show that both bean root and seed nuclear extracts contain a -213 / -175 fragment-binding activity. This activity was not been detected in nuclear extracts of bean suspension cells. This activity is strongly competed by unlabelled box III, while unlabelled box III 'double mutant' competes slightly less effectively. The nucleotide sequence in the $-213 /-175$ re- gion contains a potential SBF-1 binding site (AGTTATATG, Fig. 1) between positions -200 and -192 (inclusive) that retains six bases (underlined) of the extended SBF-1 consensus recognition site GGTTAA(A/T)(A/T)(A/T), including the central four bases of the SBF-1 core consensus recognition site GGTTAA. When compared to the box I, box II, and the two box III SBF-1 core recognition sites, this potential site shows greatest similarity to the box I core recognition site AGTTAA. In addition, we noted the presence of a sequence element (GGATGGAGATG) in the $-213 /-175$ region that is repeated in consensus (GGPuTPuGAGATG) spanning position -257 (Fig. 1). The significance of this observation is currently unknown. If the $-213 /-175$ tetramer binding activity represents SBF-1 binding to the AGTTATATG site, then GRA using equal box III-binding activities from nuclear extracts of bean cells, roots, and seeds should show equal $-213 /-175$ fragment-binding activities. This is not observed. Therefore, current data suggest that the $-213 /-175$ fragmentbinding activity is a novel protein.

Direction of GUS activity to initiating lateral roots has been described for several other $C H S$ promoters, for example, the bean $C H S 8$ promoter [28]. However, $C H S 8$ promoter activation in lateral roots persists after lateral roots have emerged from the primary root of tobacco seedlings [28], while $C H S 15$ promoter activation does not. In addition, the $C H S 8$ promoter directs expression to the lateral root meristem [28], whereas the CHS 15 promoter directs expression primarily to peripheral cell layers in a similar manner to $C H S$ transcript localization in peripheral cells of initiating pea lateral roots [31]. Furthermore, the CHS 8 promoter shows high activity in the cells of the primary root that are damaged during lateral root emergence [28], while the CHS15 promoter shows only occasional low activity in these cells. Consistent with $\mathrm{CHS} 8$ promoter activation in wounded leaves, Schmid et al. [28] have suggested that $\mathrm{CHS} 8$ promoter activity in damaged root cells represents a defense mechanism against microbial infection during emergence of the lateral roots. If so, the comparatively weak activity 
of the CHS15 promoter in these cells is consistent with our failure to observe $C H S 15$ promoter activation in wounded leaves.

GUS activity was also observed at various stages of seed development. This expression is unique to CHS15 promoter and was not observed for CHS8. Direction of GUS activity to seed epidermal cells by the CHS 15 promoter is consistent with the induction of flavonoid biosynthesis during formation of the seed coat, presumably for the synthesis of anthocyanins. GUS activity is also directed to the funicle and placenta, tissue comprising the transit regions for solute transport from parent plant to seed. This region represents a potential zone of pathogen entry into the seed line, and hence a zone where constitutive maternal expression of defense genes during seed development may be beneficial.

GUS expression is similarly directed to developing seeds by CHS promoters from other plant species. For example, the snapdragon $C H S$ gene promoter directs GUS expression in transgenic tobacco seed over a similar time course to that described here for the bean $C H S 15$ promoter, but without a tissue-specific switch in GUS activity from the endosperm to the testa [7]. In addition, both the petunia $C H S \mathrm{~A}$ and $C H S \mathrm{~J}$ promoters direct GUS reporter activity to the hexagonally shaped epidermal cells of the petunia seed testa [16] in a manner similar to bean CHS15 promoter-directed expression to the crenulated epidermal cells of the tobacco seed testa.

Why is the bean $C H S 15$ promoter activated in the tobacco endosperm? It seems unlikely that GUS activity would reflect a protective role for stress-induced gene products during early embryo development given the considerable protection offered to the plant embryo by the integuments of the seed, by the testa, and by the seed pod. Expression of GUS activity in the endosperm may reflect the proposed function of flavonoids as inhibitors of auxin transport [13]. Recently, Liu et al. [19] have shown that the establishment of a polar auxin gradient in the globular stage embryo is critical to the establishment of bilateral symmetry.

In conclusion, this study, together with previ- ous studies, have now revealed elements of fuctional architecture within the CHS15 promoter that permit organ and tissue-specific developmental patterns of regulation to be combined with flexible responses to a diverse range of environmental cues. Furthermore, this study has shown that the trans factor(s) binding to the Mae I-1 fragment of the CHS 15 promoter and the box III trimer, and the trans factor binding to the -213 / -175 tetramer, are present in nuclear extracts of bean seeds at a much higher concentration than in nuclear extracts of either bean cells or roots. This suggests a comparatively high rate of transcription of these factors in bean seeds, and hence comparatively abundant mRNA. These studies should therefore facilitate future cloning of the trans factor(s).

\section{Acknowledgements}

We thank Rick Nelson and Bruce Stermer for critical review of the manuscript and acknowledge Allyson Wilkins for assisting in the final preparation of the manuscript.

\section{References}

1. An G: Binary $\mathrm{Ti}$ vectors for plant transformation and promoter analysis. Meth Enzymol 153: 292-305 (1987).

2. Choudhary AD, Kessman H, Lamb CJ, Dixon RA: Stress responses in alfalfa (Medicago sativa L.). IV. Expression of defense gene constructs in electroporated suspension cell protoplasts. Plant Cell Rep 9: 42-46 (1990).

3. Doerner PW, Stermer B, Schmid J, Dixon RA, Lamb CJ: Plant defense gene promoter-reporter gene fusions in transgenic plants: tools for identification of novel inducers. Bio technology 8: 845-848 (1990).

4. Donald RGK, Cashmore AR: Mutation of either G or I box sequences profoundly affects expression from the Arabidopsis $r b c S-1 A$ promoter. EMBO J 9: 1717-1726 (1990).

5. Dron M, Clouse SD, Dixon RA, Lawton MA, Lamb CJ: Glutathione and fungal elicitor regulation of a plant defense gene promoter in electroporated protoplasts. Proc Natl Acad Sci USA 85: 6738-6742 (1988).

6. Firman JL, Wilson KE, Rossen L, Johnston AWB: Flavonoid activation of nodulation genes in Rhizobium reversed by other compounds present in plants. Nature 324 : 90-92 (1986). 
7. Fritze K, Staiger D, Czaja I, Walden R, Schell J, Wing D: Developmental and UV light induction of the snapdragon chalcone synthase promoter. Plant Cell 3: 893-905 (1991).

8. Green PJ, Yong M-M, Cuozzo M, Kano-Murakami Y, Silverstein $\mathrm{P}$, Chua $\mathrm{N}-\mathrm{H}$ : Binding site requirements for pea nuclear protein factor GT-1 correlate with sequences required for light-dependent transcriptional activation of the $r b c S$-3A gene. EMBO J 7: 4035-4044 (1988).

9. Hahlbrock K, Scheel D: Physiology and molecular biology of phenylpropanoid metabolism. Annu Rev Plant Physiol Mol Biol 40: 347-369 (1989).

10. Harrison MJ, Choudhary AD, Dubery I, Lamb CJ, Dixon RA: Stress responses in alfalfa (Medicago sativa L.). 8. Cis-elements and trans-acting factors for the quantitative expression of a bean chalcone synthase gene promoter in electroporated alfalfa protoplasts. Plant Mol Biol 16: 877-890 (1991).

11. Harrison MJ, Lawton MA, Lamb CJ, Dixon RA: Characterization of a nuclear protein that binds to three elements within the silencer region of a bean chalcone synthase promoter. Proc Natl Acad Sci USA 88: 2515-2519 (1991).

12. Herrera-Estrella L, Simpson J: Foreign gene expression in plants: In: Shaw $\mathrm{CH}$ (ed) Plant Molecular Biology: A Practical Approach, pp. 131-160, IRL Press, Oxford (1988).

13. Jacobs M, Rubery PH: Naturally occurring auxin transport regulators. Science 241: 346-349 (1988).

14. Jefferson RA: Assaying chimeric genes in plants: the GUS gene fusion system. Plant Mol Biol Rep 5: 387-405 (1987).

15. Jefferson RA, Kavanagh TA, Bevan MW: GUS fusions: $\beta$-glucuronidase as a sensitive and versatile gene marker in higher plants. EMBO J 6: 3901-3907 (1987).

16. Koes RE, van Blokland $R$, Quattrocchio F, van Tunen AJ, Mol JNM: Chalcone synthase promoters in petunia are active in pigmented and unpigmented cell types. Plant Cell 2: 379-392 (1990).

17. Lawton MA, Dean SM, Dron M, Kooter JM, Kragh K, Harrison MJ, Yu L, Tanguay L, Dixon RA, Lamb CJ: Silencer region of a chalcone synthase promoter contains multiple binding sites for a factor, SBF-1, closely related to GT-1. Plant Mol Biol 16: 235-249 (1991).

18. Leyva A, Liang X, Pintor-Toro JA, Dixon RA, Lamb CJ: Cis-element combinations determine phenylalanine ammonia-lyase gene tissue-specific expression patterns. Plant Cell 4: 263-271 (1992).

19. Liu C-M, Xu Z-H, Chua N-H: Auxin polar transport is essential for the establishment of bilateral symmetry during early plant embryogenesis. Plant Cell 5: 621-630 (1993).
20. Loake GJ, Choudhary AD, Harrison MJ, Mavandad M, Lamb CJ, Dixon RA: Phenylpropanoid pathway intermediates regulate transient expression of a chalcone synthase gene promoter. Plant Cell 3: 829-840 (1991).

21. Loake GJ, Factor O, Lamb CJ, Dixon RA: Combination of H-box [CCTACC(N)7CT] and G-box (CACGTG) cis elements is necessary for feed-forward stimulation of a chalcone synthase promoter by the phenylpropanoidpathway intermediate $p$-coumaric acid. Proc Natl Acad Sci USA 89: 9230-9234 (1992).

22. Peters NK, Frost JW, Long SR: A plant flavone, luteolin, induces expression of Rhizobium meliloti nodulation genes. Science 233: 977-980 (1986).

23. Redmond JW, Batley M, Djordjevic MA, Ines RW, Krempel PL, Rolfe BG: Flavones induce expression of nodulation genes in Rhizobium. Nature 323: 632-635 (1986).

24. Rogers SG, Horsch RB, Fraley RT: Gene transfer in plants: Production of transformed plants using Ti plasmid vectors. Meth Enzymol 118: 627-640 (1986).

25. Ryder TB, Hedrick SA, Bell JN, Liang X, Clouse SD, Lamb CJ: Organization and differential activation of a gene family encoding the plant defense enzyme chalcone synthase in Phaseolus vulgaris. Mol Gen Genet 210: 219223 (1987).

26. Sambrook J, Fritsch EF, Maniatis T: Molecular Cloning: A Laboratory Manual. Cold Spring Harbour Laboratory Press, Cold Spring Harbor, NY (1989).

27. Sanger F, Nicklen $S$, Coulson AR: DNA sequencing with chain-terminating inhibitors. Proc Natl Acad Sci USA 74: 5463-5467 (1977).

28. Schmid J, Doerner PW, Clouse SD, Dixon RA, Lamb CJ: Developmental and environmental regulation of a bean chalcone synthase promoter in transgenic tobacco. Plant Cell 2: 619-631 (1990).

29. Staiger D, Becker F, Schell J, Koncz C, Palme K: Purification of tobacco nuclear protein binding to a CACGTG motif of the chalcone synthase promoter by DNA affinity chromatography. Eur J Biochem 199: 519-527 (1991).

30. Stermer BA, Schmid J, Lamb CJ, Dixon RA: Infection and stress activation of bean chalcone synthase promoters in transgenic tobacco. Mol Plant-Microbe Interact 3: 381-388 (1990).

31. Yang W-C, Canter Cremers HCJ, Hogendijk P, Katinakis $P$, Wijffelman CA, Franssen H, van Kammen A, Bisseling T: In-situ localization of chalcone synthase mRNA in pea root nodule tissue. Plant J 2: 143-151 (1992).

32. Yu LM, Lamb CJ, Dixon RA: Purification and biochemical characterization of proteins which bind to the H-box cis-element implicated in transcriptional activation of plant defense genes. Plant J 3: 805-816 (1993). 